Review

D) Check for updates

\section{Diffusion Metrics as a Potential Prognostic Biomarker in Cervical Myelopathy}

\author{
Hea-Eun Yang, Seok Woo Kim, Woo-Kyoung Yoo
}

\section{HIGHLIGHTS}

Accepted: Oct 5, 2018

\section{Correspondence to}

Woo-Kyoung Yoo

Department of Physical Medicine and

Rehabilitation, Hallym University Sacred Heart

Hospital, 22 Gwanpyeong-ro 170-beon-gil,

Dongan-gu, Anyang 14068, Korea.

E-mail: mdwooky@gmail.com
- Diffusion tensor imaging (DTI) metrics are potential predictive biomarker of efficacy of surgical interventions in cervical myelopathy.

- Further efforts to make a consensus on spinal cord DTI are needed. 


\section{Review}

\section{Check for updates}

\section{OPEN ACCESS}

Received: Sep 20, 2018

Accepted: Oct 5, 2018

Correspondence to

Woo-Kyoung Yoo

Department of Physical Medicine and

Rehabilitation, Hallym University Sacred Heart

Hospital, 22 Gwanpyeong-ro 170-beon-gil,

Dongan-gu, Anyang 14068, Korea.

E-mail:mdwooky@gmail.com

Copyright (c) 2019. Korea Society for

Neurorehabilitation

This is an Open Access article distributed under the terms of the Creative Commons Attribution Non-Commercial License (https:// creativecommons.org/licenses/by-nc/4.0) which permits unrestricted non-commercial use, distribution, and reproduction in any medium, provided the original work is properly cited.

ORCID iDs

Hea-Eun Yang (D)

https://orcid.org/0000-0002-4449-7288

seok Woo Kim (iD

https://orcid.org/0000-0003-2882-4477

Woo-Kyoung Yoo (iD)

https://orcid.org/0000-0002-1273-0647

Conflict of Interest

The authors have no potential conflicts of interest to disclose.

\section{Diffusion Metrics as a Potential Prognostic Biomarker in Cervical Myelopathy}

\author{
Hea-Eun Yang $\left(\mathbb{D},{ }^{1}\right.$ Seok Woo Kim $\mathbb{1}^{2,3}{ }^{2,3}$ Woo-Kyoung Yoo (iD ${ }^{4}$ \\ 'Department of Physical Medicine and Rehabilitation, VHS Medical Center, Seoul, Korea \\ ${ }^{2}$ Spine Center, Hallym University Sacred Heart Hospital, Anyang, Korea \\ ${ }^{3}$ Department of Orthopedic Surgery, Hallym University Sacred Heart Hospital, Anyang, Korea \\ ${ }^{4}$ Department of Physical Medicine and Rehabilitation, Hallym University Sacred Heart Hospital, Anyang, Korea
}

\section{ABSTRACT}

Diffusion tensor imaging (DTI) has a diagnostic value in cervical myelopathy. The usefulness of diffusion metrics as a potential prognostic biomarker was assessed in this review. Various diffusion metrics, such as fractional anisotropy, mean diffusivity, apparent diffusion coefficient, and radial diffusivity, were significant in correlation with functional recovery after surgery. Changes in diffusion metrics not only at the compression level, but also above and below the compression level were confirmed. However, it was difficult to confirm the results owing to the diversity of studies. Further efforts to make a consensus on spinal cord DTI are needed.

Keywords: Diffusion Tensor Imaging; Cervical Myelopathy; Recovery of Function

\section{INTRODUCTION}

The incidence of cervical myelopathy, as well as degenerative cervical spinal disease, is increasing. Cervical myelopathy can be caused by compression of the spinal cord by surrounding bony or ligamentous structures and can manifest various clinical symptoms: sensory impairment and motor paralysis (from mild to complete loss) [1-3]. Decompressive surgery is the treatment of choice in symptomatic and progressive cases [4-6]. As an objective diagnostic tool, magnetic resonance imaging (MRI) is essential for the measurement of spinal cord compression and signal change. Intramedullary high signal intensities in T2-weighted (T2W) images might reflect pathological changes in the spinal cord [7-9]. However, accumulating evidence shows that high signal intensities in T2W images alone are not sensitive enough to measure the degree of injury and prognosis accurately [10]. Signal changes in T2W images often appear in the late period of clinical states and do not usually reverse even after successful decompressive surgery [9-11]. Diffusion tensor imaging (DTI), an MRI technique that is based on the diffusion properties of water molecules, has been used in quantifying structural details of the brain, especially the white matter. Recent applications of the DTI technique for the measurement of spinal cord integrity yielded a possibility of exploring the pathological condition in the spinal cord both quantitatively and qualitatively [12-14]. Currently, the use of diffusion metrics (e.g., fractional anisotropy [FA], mean diffusivity $[\mathrm{MD}]$, axial diffusivity $[\mathrm{AD}]$, and radial diffusivity [RD]) [15] and whether it 
has a clinical correlation with symptoms and prognosis are starting to draw great attention among both spinal surgeons and physiatrists. Although only a few previous studies reported on the diagnostic and prognostic values of DTI in cervical myelopathy [16-18], it is adequate for reviewing the possible usefulness of diffusion metrics in the spinal cord as a potential prognostic biomarker in longitudinal studies.

In this review, we analyzed all longitudinal studies that compared the pre-treatment and posttreatment conditions of patients with cervical myelopathy using DTI.

\section{DIFFUSION METRICS IN LONGITUDINAL STUDIES AS A POTENTIAL PROGNOSTIC BIOMARKER}

A comprehensive online search was performed to find relevant articles related to diffusion metrics as prognostic marker in cervical myelopathy. The search terms related to cervical myelopathy and DTI used were as follows: ("spinal cord," "cervical cord," "myelopathy," or "spinal cord injury") and ("diffusion magnetic resonance imaging," "diffusion tensor imaging," "diffusion MRI," or "diffusion weighted MRI"). A filter was set to restrict the search to studies with a cohort design and human studies. Among 24 initially identified articles, 7 were extracted after abstract review. Finally, we found only 3 longitudinal studies related to the predictive utility of diffusion metrics [19-21]. The other 4 studies conducted clinical followups after the treatment; however, DTI was performed only prior to the treatment [22-25]. The details are shown in Tables 1-3.

\section{Subjects}

The studies included patients with compressive myelopathy or spondylotic myelopathy who underwent surgical treatment. Compressive myelopathy was diagnosed when there were compression lesions owing to surrounding bony or ligamentous structures. Spondylotic myelopathy was included in cervical myelopathy in a broad sense.

In the 3 longitudinal studies, the average patient ages were 48 years (range: $35-82$ years), $52.8 \pm 8.0$ years, and $56.1 \pm 8.8$ years. Most of the patients showed high signal intensities on the T2W images (100\% in the study by Guan et al. [20] and 90\% in that by Yang et al. [19]). Rajasekaran et al. [21] did not describe their patients' conventional MRI findings.

In the other 4 studies that followed up clinical findings only, the subjects were slightly older than those in the longitudinal studies; their average ages were 54.5 years, $61.8 \pm 12.3$ years, 62.8 years (range: $41-82$ years), and 64 years (range: $43-86$ years). High signal intensities on the T2W images were observed in fewer patients in these 4 studies than in the longitudinal studies ( $48 \%$ in the study by Vedantam et al. [22]; 30\%, Jones et al. [23]; and 29\%, Wen et al. [25]). Arima et al. [24] did not describe their patients' conventional MRI findings.

\section{Treatments}

Surgical decompression without metallic implant was performed in 2 of the 3 longitudinal studies (laminoplasty with allo-bone graft in the study by Yang et al. [19] and anterior or posterior decompressive procedures in that by Rajasekaran et al. [21]). A titanium implant was used for anterior fixation in the study by Guan et al. [20]. Decompressive surgery was performed using surgical hardware in 2 of the 4 studies that followed up clinical findings only. The use of metal implants was one of the reasons why postoperative DTI was not 


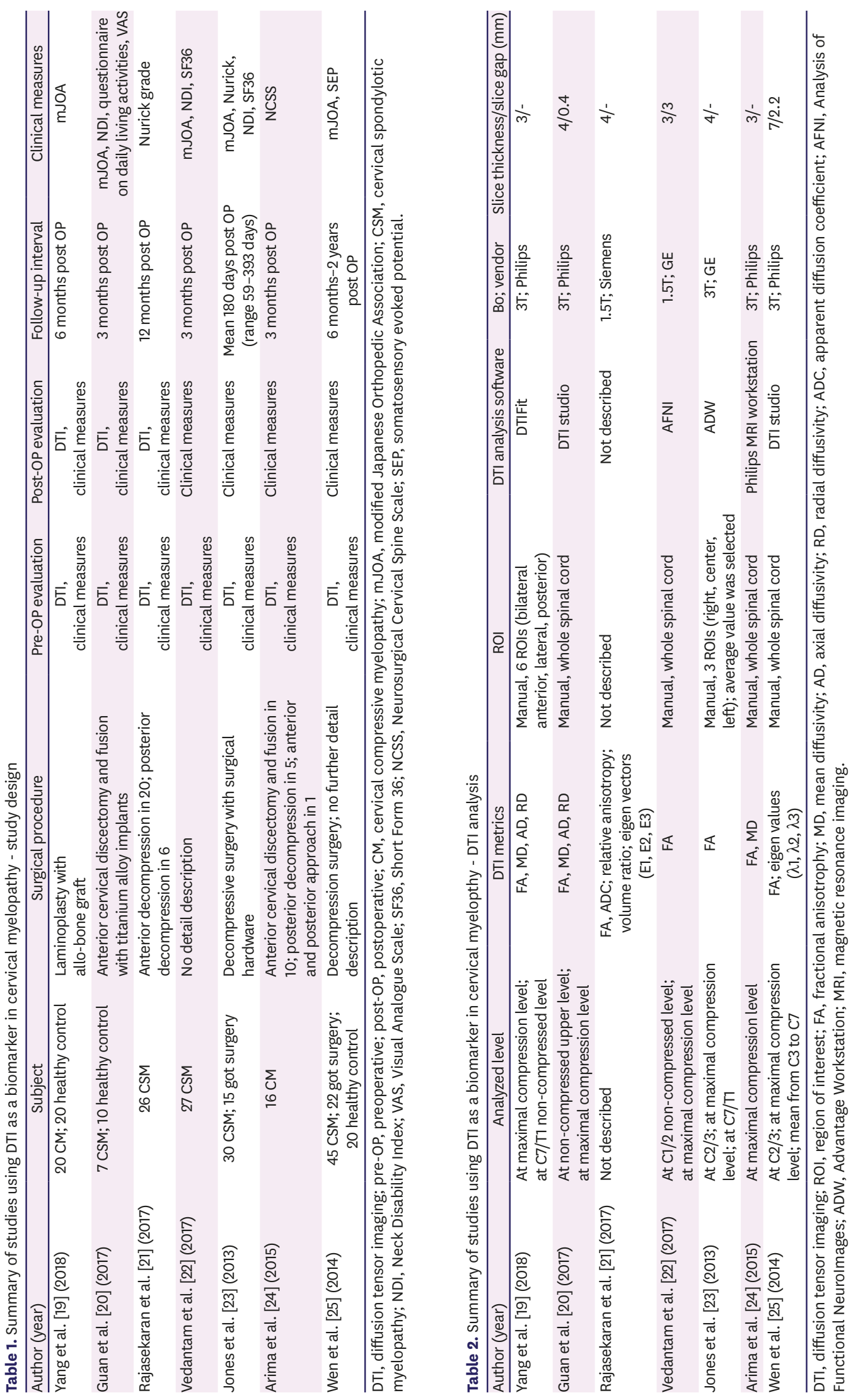


Table 3. Summary of studies using DTI as a biomarker in cervical myelopthy - acquisition and result

\begin{tabular}{|c|c|c|c|c|}
\hline Author (year) & DTI acquisition & FOV $\left(\mathrm{mm}^{2}\right)$ & TR/TE (ms) & Key results associated with recovery \\
\hline Yang et al. [19] (2018) & $\begin{array}{l}\text { Single-shot echo-planar imaging; } 15 \text { directions; } \\
b=500 \mathrm{~s} / \mathrm{mm}^{2}\end{array}$ & $250 \times 250$ & $6,300 / 63$ & $\begin{array}{l}\text { Post OP anterior FA at compression and below } \\
\text { compression level: correlate with post OP mJOA }\end{array}$ \\
\hline Guan et al. [20] (2017) & $\begin{array}{l}\text { Multi-shot, high-resolution DTI; sagittal } 6 \\
\text { directions; axial } 15 \text { directions; } b=600 \mathrm{~s} / \mathrm{mm}^{2}\end{array}$ & $250 \times 250$ & $2,500 / 72$ & $\begin{array}{l}\mathrm{FA}, \mathrm{RD} \text { at compressed region: significant differences } \\
\text { post-surgery (increased FA, decreased RD post OP) }\end{array}$ \\
\hline Rajasekaran et al. [21] (2017) & $\begin{array}{l}\text { Axial spine-echo single-shot echo planar parallel } \\
\text { imaging; } 12 \text { directions; } b=500 \mathrm{~s} / \mathrm{mm}^{2}\end{array}$ & $220 \times 220$ & $6,000 / 85$ & $\begin{array}{l}\text { Decreased in postoperative ADC, E1, E2: showed } \\
\text { neurological improvement }\end{array}$ \\
\hline Vedantam et al. [22] (2017) & $\begin{array}{l}\text { Standard single-shot, twice-refocused, spin-echo, } \\
\text { echo planar image; } 12 \text { directions; } b=600 \mathrm{~s} / \mathrm{mm}^{2}\end{array}$ & $190 \times 190$ & $5,000 / 98.2$ & $\begin{array}{l}\text { FA at max comp level: correlated with change in } \\
\text { mJOA score }\end{array}$ \\
\hline Jones et al. [23] (2013) & $\begin{array}{l}\text { Single-shot echo-planar imaging; } 6 \text { directions; } \\
b=1,000 \mathrm{~s} / \mathrm{mm}^{2}\end{array}$ & $180 \times 180$ & $8,100 / 94.1$ & Higher FA at stenosis level: NDI improve post OP \\
\hline Arima et al. [24] (2015) & $\begin{array}{l}\text { Single-shot fast-spine-echo-based sequence; } \\
15 \text { directions; } b=1,000 \mathrm{~s} / \mathrm{mm}^{2}\end{array}$ & $240 \times 240$ & $6,000 / 80$ & MD-z: correlate with functional recovery rate \\
\hline Wen et al. [25] (2014) & $\begin{array}{l}\text { Single-shot spine-echo echo-planar imaging; } \\
15 \text { directions; } b=600 \mathrm{~s} / \mathrm{mm}^{2}\end{array}$ & $80 \times 80$ & $\begin{array}{c}\text { Not } \\
\text { described }\end{array}$ & Mean FA from C3 to C7: correlate with recovery ratio \\
\hline
\end{tabular}

DTI, diffusion tensor imaging; FOV, field of view; TR/TE, repetition time/echo time; post OP, postoperative; FA, fractional anisotrophy; mJOA, modified Japanese Orthopedic Association; RD, radial diffusivity; ADC, apparent diffusion coefficient; NDI, Neck Disability Index; MD, mean diffusivity.

performed in these studies. There was no specific description of the surgical procedure in the other 2 studies.

\section{Follow-up interval}

In the studies that performed DTI after surgery, the follow-up intervals for DTI and clinical evaluation were 3 months in the study by Guan et al. [20]; 6 months, Yang et al. [19]; and 12 months, Rajasekaran et al. [21].

In the studies that performed clinical follow-ups only, the follow-up period was 3 months in 2 studies. The follow-up period was not fixed in the other 2 studies; the follow-up period ranged from 39 to 373 days in the study by Jones et al. [23] and from 6 months to 2 years in that by Wen et al. [25].

\section{Analysis of diffusion metrics Diffusion metrics}

Among the authors of the 3 longitudinal studies, Yang et al. [19] and Guan et al. [20] analyzed $\mathrm{FA}, \mathrm{MD}, \mathrm{AD}$, and RD. Rajasekaran et al. [21] analyzed FA, apparent diffusion coefficient (ADC), relative anisotropy, volume ratio, and eigen vectors. In the 4 clinical follow-up studies, FA was a common measure, and MD and eigen values were included in one study each.

Level

Among the 3 longitudinal studies, the maximal compression level and C7/T1 noncompressed level were evaluated in the study by Yang et al. [19] and maximal compression level and upper non-compressed level in that by Guan et al. [20]. The included vertebral level was not described in the study by Rajasekaran et al. [21]. In all 4 clinical follow-up studies, the maximal compression level was analyzed. Upper non-compressed areas (C1/2 and C2/3 levels) were included in 3 studies and lower non-compressed areas (C7/T1 level) in one study. One study calculated the mean FA value from $\mathrm{C} 3$ to $\mathrm{C} 7$.

\section{Region of interest (ROI)}

Only Yang et al. [19] defined 6 ROIs (i.e., bilateral anterior, lateral, and posterior) separately at each level. Jones et al. [23] defined 3 ROIs (i.e., right, left, and center) at each level; however, the average value was selected for the statistics. The ROI included the entire cord in the other studies. 


\section{DTI analysis software}

The analysis software used varied among the studies. Two studies used the DTI studio, which is an open-source diffusion MRI analysis tool [20,25]. FSL's diffusion toolkit, DTIFit, advantage workstation (AW), and analysis of functional neuroimaging processing suite were used in the other studies [19,22-37]. One study did not mention the software used for the analysis. AW is a software offered by GE healthcare, which postprocesses raw DTI data to create FA maps. All the above-mentioned software was first developed for the purpose of studying brain connectivity and activity.

\section{RESULTS}

There were 3 longitudinal studies that performed preoperative and postoperative DTI.

Yang et al. [19] analyzed FA, MD, AD, and RD at the maximal compression level and C7/ T1 non-compressed level. The postoperative FA at the compression level and below the non-compressed level correlated with the postoperative modified Japanese Orthopedic Association (mJOA) score. More specifically, FA of the anterior ROIs was correlated with recovery. This concrete result was observed because Yang et al. [19] defined 6 ROIs at each of 2 vertebral levels. The reduction of AD was not significant after surgery at the compression level and non-compressed level. Postoperative reduction in RD was noted in some ROIs; however, the changes did not correlate with recovery. There was no significant difference between the preoperative and postoperative MD [19].

Guan et al. [20] analyzed FA, MD, AD, and RD at the maximal compression level and upper non-compressed level. Postoperative increase in FA and decrease in RD at the compression level were observed in association with improvements in the visual analog scale score, mJOA score, and neck disability index. The p value was only 0.048 for RD. At the upper noncompressed level, there were no significant changes in FA and RD. There was no significant difference between the preoperative and postoperative $\mathrm{MD}$ and $\mathrm{AD}$ at the compression level and upper non-compressed level [20].

Rajasekaran et al. [21] analyzed FA, ADC, relative anisotropy, volume ratio, and eigen vectors. The included vertebral level and selection of ROIs were not described in their study. The decrease in postoperative $\mathrm{ADC}$ and eigen vectors correlated with neurological improvement after surgery. The preoperative and postoperative FA did not correlate with neurological improvement. The other diffusion metrics evaluated in their study did not show any clinical significance [21].

Taken together, the postoperative FA at the compressed level seemed to be the most sensitive biomarker for recovery after surgery in these longitudinal studies.

The 4 studies that performed clinical follow-ups after surgery conducted DTI only prior to surgery. In these studies, the correlations between preoperative diffusion metrics and postoperative recovery were studied. A higher FA value at the compression level in the preoperative period had a significant correlation with postoperative recovery in 2 studies $[22,23]$. The mean FA value from $\mathrm{C} 3$ to $\mathrm{C} 7$, not at the compression level, was correlated with recovery in one study [25]. Only Arima et al. [24] did not agree on the significance of FA; they did not use the absolute MD and FA values in their analysis and transformed the absolute values into normalized values with z-scores. A normal database from their previous study in 
30 healthy subjects was used. They concluded that a higher MD-z value was well correlated with a higher recovery rate. However, the FA-z value had no correlation with preoperative disease severity nor postoperative recovery [24].

Taken together, the preoperative FA at the compressed level seemed to be the most sensitive biomarker for recovery after surgery in these clinical follow-up studies.

\section{Limitations}

The small number of participants in each study and lack of control in some studies may weaken the validity of the results. A surgical factor might influence the DTI images obtained after surgery, especially after surgery with metal implantation. The variations in the follow-up periods might affect the comparison of the surgical outcomes. The differences in equipment, image acquisition methods, diffusion metrics, and processing across the studies pose additional challenges.

Nevertheless, the common objective of all these studies was to validate the prognostic value of diffusion metrics in cervical myelopathy. Our effort to extract valid results from these studies has a clinical value.

\section{DISCUSSION}

To date, only limited longitudinal DTI studies have been conducted; these mostly showed some correlation between diffusion metrics and prognosis in either the preoperative or postoperative period among patients with cervical myelopathy. Thus, diffusion metrics might be used as a biomarker candidate for prognostication. However, for the general use of DTI in patients with cervical myelopathy, further studies using more sophisticated mapping techniques with enhancement of acquisition protocols are warranted.

Pathophysiological changes, such as apoptotic cell death, loss of neurons, loss of oligodendrocytes, and reactive increase in astrogliosis, occur at the compression level when there are spinal cord lesions $[26,27]$. Axonal degeneration was more definite in the dorsal funiculus cranial to the compression site and in the ventral funiculus caudal to the compression site in animal models [28]. Similar microstructural changes following spinal cord injury were observed with DTI; changes in FA, generalized FA, and RD were present cranial to the lesion on the dorsal aspect and caudal to the lesion on the ventro-lateral aspect in a previous animal study [29]. The studies included in this review reported similar changes at the lesion level. Nevertheless, there is an important difference between previous animal studies and human DTI studies, which were included in this review. Only one study attempted to measure diffusion metrics separately from the ventral to the dorsal parts of the spinal cord. The remaining studies used the average value of the entire spinal cord at the lesion level; this indicates that DTI could not provide additional information than the presence of $\mathrm{T} 2$ high signal intensity in the conventional MRI except possible.

Non-compressed areas were analyzed in most of the studies included in this review. In the 4 studies that performed DTI in the cranial non-compressed areas, none of the diffusion metrics from the cranial to the compression levels were significant in predicting recovery $[20,21,23,25]$. However, in one study, the preoperative FA above the lesion had a strong correlation with the baseline functional scores [23]. Of the 2 studies that performed DTI in the caudal non-compressed areas, only the study by Yang et al. [19] showed a significance 
in the FA value below the lesion level. It is plausible to propose that measuring the motor tract below the lesion level would have a clinical importance as Wallerian degeneration can be present, which can change the diffusion metrics below the lesion level. Another study concluded that there is no correlation between the FA value below the lesion and recovery, which may be because the ROI was defined to cover the entire spinal cord and the anatomical portion of the tracts was not reflected [23].

The surgical intervention changed the diffusion metrics in all 3 longitudinal studies, which might indicate the possibility of using them as a biomarker. The FA value showed a clear correlation with postoperative recovery in all 3 studies. These results support the proposition that microstructural changes occurring after surgical treatment measured by FA clearly reflect the functional outcomes. This result is in line with previous evidence that the FA value is well correlated with its function in other diseases [12,30-32].

Among the 3 longitudinal studies, only 2 studies measured the MD, $\mathrm{AD}$, and $\mathrm{RD}$ values. One study did not analyze the diffusion metrics, except for the FA value, which would not be enough to delineate underlying pathological states.

$\mathrm{MD}$ is an inverse measure of membrane density and is sensitive to cellularity, edema, and necrosis [33]. Its value is the sum of the $\mathrm{AD}$ and $\mathrm{RD}$ values [34]. The $\mathrm{AD}$ value tends to be variable in white matter changes and pathologies and decreases in axonal injuries [34,35], while the $\mathrm{RD}$ value increases in white matter changes with demyelination or dysmyelination. Changes in axonal diameters or density may also influence $\mathrm{RD}[33,36]$. In 2 studies, the $\mathrm{MD}$ and $\mathrm{AD}$ values did not change significantly after surgery. Only the RD value was reversed after surgery in one study; in this study, the authors interpreted this result as reversed myelinopathy after surgery. The result variations between the 2 studies may be because one study used metallic device during surgery, causing severe artifact. If we exclude this study for possible artifact noise, then the other study would have reported recovery after surgery with plausible pathological changes.

Notably, recent advances in spinal cord imaging tools, such as the Spinal Cord Toolbox (NeuroPoly, École Polytechnique, Université de Montréal campus, Montreal, Canada), may increase the scientific analytical methods using sophisticated acquisition and mapping techniques in the spinal cord.

The detailed analysis process was described elsewhere [37]. As this technique uses meticulous segmentation and registration algorithm specific to the spinal cord, it can provide a good solution for the above-mentioned limitations. There are many physiological and motion artifacts in spinal cord imaging owing to spinal cord pulsation, cardiac pulsation, and respiration; preparing optimal acquisition protocols by reducing field of view approaches (2-dimensional radiofrequency excitation, outer-volume suppression methods, and parallel imaging) [36] would be essential in obtaining reliable scientific data, instead of employing the entire spinal cord as a single ROI or 6 ROIs manually.

\section{CONCLUSION}

DTI FA is a potential predictive biomarker of efficacy of surgical interventions in cervical myelopathy. The prognostic value of other diffusion metrics need to be assessed through more structured studies. Further efforts to make a consensus on spinal cord DTI are needed. 


\section{REFERENCES}

1. Fehlings MG, Skaf G. A review of the pathophysiology of cervical spondylotic myelopathy with insights for potential novel mechanisms drawn from traumatic spinal cord injury. Spine (Phila Pa 1976) 1998;23:2730-2737. PUBMED | CROSSREF

2. Pepke W, Almansour H, Richter M, Akbar M. Spondylotic cervical myelopathy: indication of surgical treatment. Orthopade 2018;47:474-482. PUBMED | CROSSREF

3. Hasegawa K, Homma T, Chiba Y, Hirano T, Watanabe K, Yamazaki A. Effects of surgical treatment for cervical spondylotic myelopathy in patients $>$ or $=70$ years of age: a retrospective comparative study. $\mathrm{J}$ Spinal Disord Tech 2002;15:458-460. PUBMED | CROSSREF

4. Chikuda H, Ohtsu H, Ogata T, Sugita S, Sumitani M, Koyama Y, Matsumoto M, Toyama Y; OSCIS investigators. Optimal treatment for spinal cord injury associated with cervical canal stenosis (OSCIS): a study protocol for a randomized controlled trial comparing early versus delayed surgery. Trials 2013;14:245. PUBMED | CROSSREF

5. Payer M, Hodler J, Benini A. Surgical treatment of cervical myelopathy of unclear aetiology. J Clin Neurosci 2004;11:159-162. PUBMED | CROSSREF

6. Sumi M, Miyamoto H, Suzuki T, Kaneyama S, Kanatani T, Uno K. Prospective cohort study of mild cervical spondylotic myelopathy without surgical treatment. J Neurosurg Spine 2012;16:8-14. PUBMED | CROSSREF

7. Matsumoto M, Toyama Y, Ishikawa M, Chiba K, Suzuki N, Fujimura Y. Increased signal intensity of the spinal cord on magnetic resonance images in cervical compressive myelopathy. Does it predict the outcome of conservative treatment? Spine (Phila Pa 1976) 2000;25:677-682. PUBMED | CROSSREF

8. Li F, Chen Z, Zhang F, Shen H, Hou T. A meta-analysis showing that high signal intensity on T2-weighted MRI is associated with poor prognosis for patients with cervical spondylotic myelopathy. J Clin Neurosci 2011;18:1592-1595. PUBMED | CROSSREF

9. Zhang YZ, Wang LF, Shen Y, Ding WY, Xu JX, He J. The effects of MRI signal intensity changes and clinical manifestations on prognosis after surgical intervention for cervical spondylotic myelopathy. Orthop Surg 2009;1:101-106.

PUBMED | CROSSREF

10. Yang YM, Yoo WK, Yoo JH, Kwak YH, Oh JK, Song JS, Kim SW. The functional relevance of diffusion tensor imaging in comparison to conventional MRI in patients with cervical compressive myelopathy. Skeletal Radiol 2017;46:1477-1486. PUBMED | CROSSREF

11. Yonenobu K. Cervical radiculopathy and myelopathy: when and what can surgery contribute to treatment? Eur Spine J 2000;9:1-7. PUBMED | CROSSREF

12. Song T, Chen WJ, Yang B, Zhao HP, Huang JW, Cai MJ, Dong TF, Li TS. Diffusion tensor imaging in the cervical spinal cord. Eur Spine J 2011;20:422-428. PUBMED | CROSSREF

13. Gao SJ, Yuan X, Jiang XY, Liu XX, Liu XP, Wang YF, Cao JB, Bai LN, Xu K. Correlation study of 3T-MR-DTI measurements and clinical symptoms of cervical spondylotic myelopathy. Eur J Radiol 2013;82:1940-1945. PUBMED | CROSSREF

14. Kara B, Celik A, Karadereler S, Ulusoy L, Ganiyusufoglu K, Onat L, Mutlu A, Ornek I, Sirvanci M, Hamzaoglu A. The role of DTI in early detection of cervical spondylotic myelopathy: a preliminary study with 3-T MRI. Neuroradiology 2011;53:609-616. PUBMED | CROSSREF

15. Cohen-Adad J, El Mendili MM, Lehéricy S, Pradat PF, Blancho S, Rossignol S, Benali H. Demyelination and degeneration in the injured human spinal cord detected with diffusion and magnetization transfer MRI. Neuroimage 2011;55:1024-1033. PUBMED | CROSSREF

16. Yoo WK, Kim TH, Hai DM, Sundaram S, Yang YM, Park MS, Kim YC, Kwak YH, Ohn SH, Kim SW. Correlation of magnetic resonance diffusion tensor imaging and clinical findings of cervical myelopathy. Spine J 2013;13:867-876. PUBMED | CROSSREF 
17. Xiangshui M, Xiangjun C, Xiaoming Z, Qingshi Z, Yi C, Chuanqiang Q, Xiangxing M, Chuanfu L, Jinwen H. 3 T magnetic resonance diffusion tensor imaging and fibre tracking in cervical myelopathy. Clin Radiol 2010;65:465-473. PUBMED | CROSSREF

18. Yildiz S, Cece H, Turksoy O. RE: 3 T magnetic resonance diffusion tensor imaging and fibre tracking in cervical myelopathy. Clin Radiol 2011;66:390-391.

PUBMED | CROSSREF

19. Yang YM, Yoo WK, Bashir S, Oh JK, Kwak YH, Kim SW. Spinal cord changes after laminoplasty in cervical compressive myelopathy: a diffusion tensor imaging study. Front Neurol 2018;9:696. PUBMED | CROSSREF

20. Guan L, Chen X, Hai Y, Ma X, He L, Wang G, Yuan C, Guo H. High-resolution diffusion tensor imaging in cervical spondylotic myelopathy: a preliminary follow-up study. NMR Biomed 2017;30:30. PUBMED | CROSSREF

21. Rajasekaran S, Kanna RM, Chittode VS, Maheswaran A, Aiyer SN, Shetty AP. Efficacy of diffusion tensor imaging indices in assessing postoperative neural recovery in cervical spondylotic myelopathy. Spine (Phila Pa 1976) 2017;42:8-13. PUBMED | CROSSREF

22. Vedantam A, Rao A, Kurpad SN, Jirjis MB, Eckardt G, Schmit BD, Wang MC. Diffusion tensor imaging correlates with short-term myelopathy outcome in patients with cervical spondylotic myelopathy. World Neurosurg 2017;97:489-494. PUBMED | CROSSREF

23. Jones JG, Cen SY, Lebel RM, Hsieh PC, Law M. Diffusion tensor imaging correlates with the clinical assessment of disease severity in cervical spondylotic myelopathy and predicts outcome following surgery. AJNR Am J Neuroradiol 2013;34:471-478.

PUBMED | CROSSREF

24. Arima H, Sakamoto S, Naito K, Yamagata T, Uda T, Ohata K, Takami T. Prediction of the efficacy of surgical intervention in patients with cervical myelopathy by using diffusion tensor 3T-magnetic resonance imaging parameters. J Craniovertebr Junction Spine 2015;6:120-124. PUBMED | CROSSREF

25. Wen CY, Cui JL, Liu HS, Mak KC, Cheung WY, Luk KD, Hu Y. Is diffusion anisotropy a biomarker for disease severity and surgical prognosis of cervical spondylotic myelopathy? Radiology 2014;270:197-204. PUBMED | CROSSREF

26. Yu WR, Baptiste DC, Liu T, Odrobina E, Stanisz GJ, Fehlings MG. Molecular mechanisms of spinal cord dysfunction and cell death in the spinal hyperostotic mouse: implications for the pathophysiology of human cervical spondylotic myelopathy. Neurobiol Dis 2009;33:149-163. PUBMED | CROSSREF

27. Carlson GD, Gorden CD, Oliff HS, Pillai JJ, LaManna JC. Sustained spinal cord compression, part I: timedependent effect on long-term pathophysiology. J Bone Joint Surg Am 2003;85-A:86-94. PUBMED | CROSSREF

28. Yoon H, Kim J, Moon WJ, Nahm SS, Zhao J, Kim HM, Eom K. Characterization of chronic axonal degeneration using diffusion tensor imaging in canine spinal cord injury: a quantitative analysis of diffusion tensor imaging parameters according to histopathological differences. J Neurotrauma 2017;34:3041-3050. PUBMED | CROSSREF

29. Cohen-Adad J, Leblond H, Delivet-Mongrain H, Martinez M, Benali H, Rossignol S. Wallerian degeneration after spinal cord lesions in cats detected with diffusion tensor imaging. Neuroimage 2011;57:1068-1076. PUBMED | CROSSREF

30. Budzik JF, Balbi V, Le Thuc V, Duhamel A, Assaker R, Cotten A. Diffusion tensor imaging and fibre tracking in cervical spondylotic myelopathy. Eur Radiol 2011;21:426-433. PUBMED | CROSSREF

31. Mamata H, Jolesz FA, Maier SE. Apparent diffusion coefficient and fractional anisotropy in spinal cord: age and cervical spondylosis-related changes. J Magn Reson Imaging 2005;22:38-43. PUBMED | CROSSREF

32. Basser PJ, Pierpaoli C. Microstructural and physiological features of tissues elucidated by quantitativediffusion-tensor MRI. J Magn Reson B 1996;111:209-219. PUBMED | CROSSREF

33. Chung MK, Hanson JL, Adluru N, Alexander AL, Davidson RJ, Pollak SD. Integrative structural brain network analysis in diffusion tensor imaging. Brain Connect 2017;7:331-346. PUBMED | CROSSREF 
34. Alexander AL, Hurley SA, Samsonov AA, Adluru N, Hosseinbor AP, Mossahebi P, Tromp PM, Zakszewski E, Field AS. Characterization of cerebral white matter properties using quantitative magnetic resonance imaging stains. Brain Connect 2011;1:423-446. PUBMED | CROSSREF

35. Alexander AL, Lee JE, Lazar M, Field AS. Diffusion tensor imaging of the brain. Neurotherapeutics 2007;4:316-329. PUBMED | CROSSREF

36. Cohen Y, Anaby D, Morozov D. Diffusion MRI of the spinal cord: from structural studies to pathology. NMR Biomed 2017;30:30. PUBMED | CROSSREF

37. Cohen-Adad J. Functional magnetic resonance imaging of the spinal cord: current status and future developments. Semin Ultrasound CT MR 2017;38:176-186.

PUBMED | CROSSREF 